\title{
Conocimiento sobre consentimiento informado de médicos en formación
}

\author{
Knowledge on informed consent by in training physicians
}

\author{
Salomón Zavala ${ }^{1}$, Cecilia Sogi ${ }^{1}$, Ana Delgado ${ }^{1}$, Maximiliano Cárdenas ${ }^{2}$ \\ 1 Instituto de Ética en Salud, Facultad de Medicina, Universidad Nacional Mayor de San Marcos. Lima, Perú. \\ ${ }^{2}$ Departamento Académico de Cirugía, Facultad de Medicina, Universidad Nacional Mayor de San Marcos. Lima, Perú.
}

\begin{abstract}
Resumen
Objetivo: Evaluar qué saben sobre consentimiento informado los postulantes a la residencia médica. Diseño: Cuestionario sobre consentimiento informado. Institución: Facultad de Medicina, Universidad Nacional Mayor de San Marcos. Participantes: Médicos postulantes al examen de admisión a la residencia médica. Metodología: Se elaboró un examen de conocimiento con preguntas de elección múltiple. Participaron en el estudio 1486 (95\%) postulantes a las plazas de residencia médica de la Universidad Nacional Mayor de San Marcos, 2009. Se elaboró una base de datos en Excel y se utilizó el paquete estadístico SPSS 15 para análisis de frecuencia; y Atlas ti para análisis cualitativo. La participación en el estudio fue voluntaria y anónima. Principales medidas de resultados: Conocimiento sobre consentimiento informado. Resultados: De los 1486 participantes, 56\% fue varón, con edad media de 31,5 (DE 5,1) años, rango entre 23 y 58 años, egresados de 26 escuelas de medicina del país y 14 del exterior; uno de cada tres egresó entre 2007 y 2008. La gran mayoría (84\%) respondió que respetar la autonomía del paciente significa mejorar la comunicación médico paciente; y tres de cada cuatro, que obtener el consentimiento informado es un imperativo ético. La frecuencia de aciertos disminuyó en las preguntas sobre información sobre quién determina la capacidad mental y qué hacer cuando un paciente rechaza tratamiento. Conclusiones: La población de estudio fue heterogénea en edad, universidad de origen y año de egreso. Se identificó deficiencias en conocimiento del consentimiento informado.

Palabras clave: Consentimiento informado; conocimiento; evaluación; estudiantes de medicina.
\end{abstract}

\begin{abstract}
Objectives: To determine physicians applicants to the medical residency's knowledge on informed consent. Design: Questionnaire on informed consent. Setting: Faculty of Medicine, Universidad Nacional Mayor de San Marcos. Participants: Physicians applying to postgraduate medical residency. Methods: A multiple choice examination was elaborated. One thousand four hundred and eighty six (95\%) applicants to the Universidad Nacional Mayor de San Marcos 2009 medical residency participated in the study. An Excel data base was prepared and SPSS 15 for statistic frequency analysis and Atlas-ti for qualitative analysis were used. Participation in the study was voluntary and anonymous. Main outcome measures: Knowledge on informed consent. Results: Fifty-six per cent of participants were male with a mean age of 31.5 (S.D. 5.1) and range between 23 and 58. They graduated in 26 national medical schools and 14 foreign medical schools; one of every three graduated between 2007 and 2008. The majority (84\%) answered that to respect patient's autonomy improves doctor/patient communication; three of every four stated that to obtain informed consent is an ethical imperative. The frequency of correct answers decreased with questions on who establishes the mental capacity and what to do when the patient refuses treatment. Conclusions: The studied population was heterogeneous with regards to age, university of origin and year of graduation. Some deficiencies on knowledge on informed consent were identified.

Key words: Informed consent; knowledge; evaluation; students, medical.
\end{abstract}

\section{INTRODUCCIÓN}

El consentimiento informado es el instrumento mediante el cual el paciente ejerce su derecho a la autodeterminación y para ello el médico está en el deber de dar la información que aquel necesita. El requerimiento de obtener el consentimiento ha sido ampliamente fundamentado en un replanteamiento de la relación médico paciente tradicional, sustentado en el reconocimiento de la autonomía individual.

Sobre los orígenes del consentimiento informado, el modelo paternalista de relación que caracterizó a la práctica médica, desde sus orígenes, ha cedido paso a uno nuevo, el consentimiento informado. Esa relación, que data de la época de oro de la medicina griega hace 25 siglos atrás, está cediendo paso a propuestas de modelos de relación más horizontales.
La particular relación médico paciente de inicios de la medicina occidental tiene su sustrato racional, tal como la relata Sánchez García: “... el médico deja los astros para posarse en el enfermo y la enfermedad se ubica en el cuerpo del hombre. Si antes el corazón y el hígado de los animales sacrificados para los presagios eran fundamentales herramientas del diagnóstico y el pronóstico, el hipocratismo hace que el médico se interese por esos órganos en el hombre. Por primera, vez el órgano y el enfermo van de la mano en el drama de la enfermedad; el enfermo se entrega al médico que concibe la enfermedad como algo concreto en el hombre y no en el cosmos..." (1).

Las características de la relación paternalista lo ilustra el siguiente texto hipocrático sobre la decencia citado por Diego Gracia (2): "Haz todo esto (el diagnóstico y tratamiento del enfermo) con calma y orden, ocultando al enfermo, durante tu actuación, la mayoría de las cosas. Dale las órdenes oportunas con amabilidad y dulzura y distrae su atención; repréndelo a veces estricta y severamente, pero otras, anímale con solicitud y habilidad, sin mostrarle nada de lo que le va a pasar ni de su estado actual; pues muchos acuden a otros médicos por causa de esa declaración, antes mencionada, del pronóstico sobre su presente y futuro".

En la historia de los orígenes del consentimiento, Simón describe cómo, en los últimos siglos, la modernidad introduce la idea de que: "en principio, todas las personas tienen total autonomía moral para decidir en las cuestiones relevantes para su proyecto vital" (3).

El consentimiento como ejercicio del respeto a la autonomía nunca ha tenido 
un sustrato firme en la práctica médica. Faden y Beauchamp, en una revisión sistemática del tema, dicen que esta ha sido siempre una idea ajena a los escritos influyentes en la historia de la medicina y la ética médica. De mayor peso fueron los principios hipocráticos y percivalianos, que los médicos no deben perjudicar a pacientes revelándoles muy abrupta y crudamente sobre su condición, apelando a un modelo de beneficencia médica ${ }^{(4)}$.

El término consentimiento informado y el desarrollo de su teoría legal tuvieron lugar en los Estados Unidos. Faden y Beauchamp atribuyen este fenómeno a factores externos a la medicina, "El creciente interés en el consentimiento informado en la atención médica de mediados del siglo XX pudo ser resultado de complejas fuerzas sociales que cambiaron el estatus y el rol de la medicina norteamericana, como una reacción frente a desarrollos legales específicos. Un ejemplo claro lo hallamos en la Carta de derechos del paciente de la Asociación americana de hospitales, de 1972. Esto fue, en gran parte, resultado de la presión del consumidor por una mejor atención, así como por un estándar de respeto más adecuado. Este mayor respeto se hace manifiesto a través del reconocimiento de la necesidad de obtener el consentimiento informado y respetar la negativa al tratamiento médico" (4).

Respecto a la doctrina legal del consentimiento informado en los EEUU, Faden y Beauchamp señalan que el consentimiento informado se interpreta en la tradición legal como basado en, y justificado por, el principio de respeto a la autonomía. Sin embargo, en el contexto legal, el consentimiento no es precisamente acerca de cómo respetar mejor la autonomía del paciente o permitirle una toma de decisión autónoma. El lenguaje legal se orienta hacia derechos y deberes específicos (correlativos) que derivan de los principios. Así, en caso legal, la justificación del consentimiento se expresa en lenguaje de derecho -el derecho del paciente a la autodeterminación- y el interés primario de la ley en prescribir los deberes que corresponden a los médicos a fin de que se proteja este derecho ${ }^{(4)}$.

En 1947 salió a la luz el Código de Nuremberg, como resultado del juicio al que fueron sometidos muchos de los médicos nazis que experimentaron con prisioneros de guerra durante la Segunda Guerra Mundial. Su primera norma se refiere al consentimiento voluntario de los sujetos de estudio. Vargas-Parada y col. citan un documento predecesor titulado "Lineamientos para la terapia innovadora y experimentos científicos en el hombre", publicado el 28 de febrero de 1931, por el Ministerio del Interior alemán ${ }^{(5)}$.

El informe Belmont (Ethical principles and guidelines for the protection of human subjects of research), publicado en 1978, en los Estados Unidos, trata de regular toda la investigación biomédica mediante criterios muy generales. Parte del Código de Nuremberg y de la Declaración de Helsinki (1964) ${ }^{(6)}$.

Otros acercamientos fueron Making Health Care Decisions. The Ethical and Legal Implications of Informed Consent in the Patient-Practitioner Relationship (President's Commission for the Study of Ethical Problems in Medicine and Biomedical and Behavioral Research). Siguiendo el método de la National Commission, este comité se abocó a los problemas morales planteados por el ejercicio de la biomedicina moderna. Esta señala que: "La fundamentación ética del consentimiento informado puede encontrarse en el intento de promover dos valores: el bienestar personal y la autodeterminación. Para garantizar que estos valores sean respetados y realzados, la Comisión declara que a los pacientes que tengan capacidad para tomar decisiones acerca de su cuidado debe permitírseles hacerlo de forma voluntaria y se les debe proporcionar toda información relevante acerca de su situación y de los tratamientos alternativos, incluyendo beneficios posibles, riesgos, costes, otras consecuencias previsibles y el grado significativo de incertidumbre que atañe a cualquiera de estas informaciones" (7).

El consentimiento informado hoy en día ha sido introducido en la práctica médica mundial a través de leyes (como resultado de casos judiciales) y códigos de colegios profesionales. En España, dice Simón Lorda, el paternalismo tradicional en las relaciones sanitarias empieza a cambiar a mediados de la década del 80, con la aparición de la primera Carta de derechos de los pacientes, en el artículo 4 de la Ley General de Sanidad, paralelamente al ingreso de la bioética en diferentes sectores sociales y la consciencia cada vez mayor de los derechos ciudadanos ${ }^{(3)}$.

En el Perú, el consentimiento informado $(\mathrm{CI})$ se introduce con la Ley General de Salud ( $N^{\circ}$ 26842), vigente desde julio de 1997; en su artículo 4 establece la obligatoriedad del CI en la práctica médica ${ }^{(8)}$. Su reglamento establece los casos y requisitos de formalidad que deben ser observados para que el CI sea considerado válidamente emitido. El 2 de octubre del 2009 se publica modificaciones a esta ley: en sus artículos 15.2, acceso a la información; 15.3, atención y recuperación de la salud, y 15.4, consentimiento informado (9). Paralelamente, el Código de ética y deontología del Colegio Médico del Perú, en sus sucesivas revisiones, contempla, bajo el título de relación médico paciente, la necesidad del consentimiento en la práctica médica ${ }^{(10)}$.

En relación a los elementos o requisitos del consentimiento válido en la práctica clínica, el consenso señala que: a) el médico debe proporcionar información adecuada al paciente; b) no tiene que coaccionar al paciente para que consienta en el tratamiento; y, c) debe asegurarse de que el paciente sea competente para dar su consentimiento o rechazar un tratamiento $^{(11)}$.

En un estudio empírico de los elementos de la decisión informada en grabaciones de diálogo médico paciente, Braddock y col. hallaron que apenas el 9\% alcanzó la definición de toma de decisión informada, y menos de 10\% de los médicos discutió con el paciente acerca del tratamiento, alternativas, riesgos, incertidumbre ${ }^{(12)}$. Y en una encuesta en 3000 médicos graduados, entre 1974. 1978, Pellegrino y col. hallaron que la obtención del consentimiento informado ocupaba el cuarto lugar entre los problemas éticos más frecuentes en la práctica clínica, siendo los tres primeros: comprensión, veracidad en la información y participación del paciente en la toma de decisión ${ }^{(13)}$. 
El desarrollo de la teoría del consentimiento informado en Derecho y su introducción a la práctica clínica y en la investigación biomédica es un tema central en la educación ética del médico. Las razones serían porque el consentimiento informado: a) es el modelo de relación médico paciente ideal en los tiempos actuales, en los términos de Diego Gracia; $y, b)$ constituye un imperativo ético en la práctica médica y en investigación.

Paralelamente a lo mencionado, en las últimas décadas, las escuelas de medicina vienen incorporando la educación ética en su programa de estudios de pre y posgrado y, hoy en día, los estándares de acreditación de tales programas señalan explícitamente la necesidad de una educación ética del médico.

Respecto a la enseñanza ética en las escuelas de medicina, existen esfuerzos de consenso para su óptima puesta en marcha tales como las de Culver y colaboradores en los Estados Unidos, Basic curricular goals in medical ethics (1985) ${ }^{(14)}$; Consensus statement by teachers of medical ethics and law in UK (1998) ${ }^{(15)}$; y Working group on behalf of the Association of Teachers of Ethics and Law in Australian and New Zealand Medical Schools (2000) ${ }^{(16)}$.

En el Perú, la ética es parte de plan de estudios médicos desde la década de los 80s. Un análisis preliminar de un estudio en curso halló que la mayoría de las escuelas incluye el tema del consentimiento informado en su curso de ética; sin embargo, otro estudio en internos de medicina de una universidad pública capitalina halló deficiencias de formación en el tema ${ }^{(17)}$. A ello se agrega la observación anecdótica de que en nuestro medio no existe una comprensión cabal de qué es el consentimiento informado y por qué la importancia de obtenerla en la práctica clínica $^{(18)}$.

Para evaluar la enseñanza, se ha desarrollado instrumentos que evalúan la conciencia ética, como el Toronto Ethical Sensitivity Instrument, que mide específicamente problemas éticos identificados en viñetas de casos. Otros evalúan cambios en el razonamiento moral, Kohlberg's Moral Judgement Interview y el Sociomoral Reasoning Measure. El Objective Structured
Clinical examination (OSCE) determina la habilidad del entrevistado para actuar éticamente en una situación clínica ${ }^{(19)}$.

De los pocos estudios sobre el tema de evaluación del conocimiento sobre el consentimiento está el de Fisher-Jeffes y col. Los autores utilizaron un cuestionario con 10 viñetas breves de casos pediátricos, pidiendo que respondieran con verdadero, falso o no sé. Participaron 51 médicos de diferentes especialidades. Los pediatras alcanzaron puntajes mayores que el resto de especialistas (puntaje promedio de respuestas correctas $96 \%$ versus $49 \%)^{(20)}$.

Skene y Smallwood evaluaron el conocimiento legal sobre el consentimiento. Enviaron cuestionarios a 950 médicos de Victoria y Tasmania y respondieron 246 (25\%). Menos de la mitad de los participantes reconoció que el Dr. Roger fue negligente por no informar sobre el riesgo de 1:14 000 de oftalmia simpática y tuvo que pagar por daños. Cerca de $40 \%$ respondió que tenía que informar a los pacientes todos los riesgos o resultados posibles de la intervención, pero el porcentaje restante fue reticente a dar información. Aparentemente, muchos médicos no dan información y se apoyan en bases cuestionables para ocultar información (condición física del paciente o inestabilidad emocional) ${ }^{(21)}$.

En estudiantes de medicina y residentes de la Universidad de Nueva México, Roberts y col. hallaron que los participantes manifestaron claramente su interés a que se preste mayor atención en su formación a tópicos relacionados con el consentimiento, tales como, obtener consentimiento de personas incapaces de tomar decisiones, decisión sustituta, que no hablan inglés y los que rechazan el tratamiento recomendado, entre otros tópicos ${ }^{(22)}$.

Utilizando dos viñetas de casos, Bremberg y Nilstun evaluaron el razonamiento ético de médicos generales noruegos. Se les pidió que respondieran cómo deberían actuar y cómo actuarían cuando el derecho del paciente a la autodeterminación entra en conflicto con la obligación médica de promover el bienestar. Frente a pacientes renuentes a aceptar una in- tervención médica, la mayoría respondió que el derecho a la autodeterminación debe ser el factor predominante. Frente a un paciente que solicita una intervención médicamente dudosa, hubo mayor concordancia entre valores y práctica. La mayoría respondió que no accederá a la petición del paciente ${ }^{(23)}$.

El objetivo general del presente estudio fue evaluar el conocimiento sobre consentimiento informado de médicos en formación (postulantes a la residencia), siendo el propósito mejorar el proceso enseñanza aprendizaje del tema, más que cuantificar el número de aciertos.

\section{MÉTODOS}

Los participantes en el estudio fueron 1486 médicos ( $95 \%$ del total) postulantes al examen de admisión a la residencia médica de la Universidad Nacional Mayor de San Marcos (UNMSM) 2009.

El borrador del examen de conocimiento sobre el CI fue elaborado por dos investigadores (CS y SZ). El examen comprende nueve preguntas estructuradas para elegir una de cuatro opciones de respuesta. Al final de las preguntas se proporciona un espacio para comentarios y, por último, se pidió anotar edad, sexo, universidad donde estudió medicina y el año en que terminó la carrera.

Las preguntas 1 y 2 exploraban el objetivo del consentimiento informado. La 3, 7 y 8 remiten a algunas características de la información que el médico está obligado a proporcionar en el proceso de obtención del CI. La 4 y la 9 examinaban la toma de decisión en el proceso del consentimiento. La pregunta 5 era conceptual, sobre el fundamento del consentimiento. La 6 era una pregunta práctica que exploraba quién determina la capacidad mental (competencia) del paciente en la atención de salud.

Se solicitó autorización al Director de la Unidad de Postgrado, Facultad de Medicina, UNMSM, para la aplicación del cuestionario. Luego de su aprobación, la recolección de datos se coordinó con el jefe de la sección de Segunda Especialización. Esta tuvo lugar el 25 de mayo de 2009. 
La validez del contenido se estableció a través de la prueba de jueces. Participaron ocho miembros del Instituto de Ética en Salud, de la UNMSM. Se redactó un cuestionario que indagaba si las preguntas y opciones de respuesta eran claras. Los ocho jueces contribuyeron con importantes sugerencias para mejorar el instrumento. Uno de ellos sugirió: "En general habría que precisar mejor las preguntas y tener en cuenta el contexto clínico y legal en nuestro medio, y tender a que las respuestas sean unívocas".

La concordancia entre jueces se estableció mediante la prueba propuesta por Aiken ${ }^{(24)}$. Esta fue significativa $(p<0,05)$ en cinco preguntas y no significativa $(p>0,05)$ en las restantes cinco. Las últimas fueron revisadas para incorporar las sugerencias de los jueces, tanto en el fraseo de las preguntas como en las opciones de respuesta.

Para la confiabilidad del instrumento y no contando con una población similar previo al trabajo de campo, se utilizó los resultados del examen con los 1486 participantes. Se aplicó la prueba alfa de Cronbach y la confiabilidad fue de 0,235 .

Para la recolección de datos, el instrumento fue aplicado el 25 de mayo de 2009, en el local central de la Facultad de Medicina, luego de concluido el examen de admisión a la residencia médica.

Los comentarios fueron transcritos verbatim y luego clasificados en categorías. Se revisó los cuestionarios, separándose aquellos incompletos. Luego, se elaboró una base de datos en EXCEL. Se utilizó el paquete estadístico SPSS versión 15 para análisis descriptivo y el Atlas ti para análisis cualitativo.

Se comunicó verbalmente a los postulantes que la participación era voluntaria, que el examen era anónimo y que los datos serían utilizados solo para los propósitos del presente estudio.

\section{RESULTADOS}

Participaron en el estudio 1486 médicos. El 56\% fue varón. La edad promedio fue 31,5 (DE 5,1), edad mínima 23 y máxima 58. El rango de edad, 29 años 38,5\%; 30 a
34 años, 39,8\%; 35 a 39 años, $15,5 \%$; y 40 o más años, 6,5\%. Cuatro de cada cinco participantes fue menor de 35 años.

Los participantes procedían de 26 escuelas de medicina del país y 14 del exterior. El mayor porcentaje, 21,5\%, fue egresado de UNMSM, seguido de universidades nacionales de provincias: San Luis Gonzaga, Ica (7,0\%), San Antonio Abad, Cusco (4,8\%), San Agustín, Arequipa (4,8\%) y Trujillo (4,0\%); y de universidades particulares de Lima, San Martín de Porres (3,6\%), y de provincias, Católica Santa María, Arequipa (2,6\%).

El total de participantes egresó entre 1982 y 2008, siendo el año de egreso promedio 2004.

Nuestro estudio de evaluación del conocimiento sobre consentimiento informado exploró lo que sabían más que cuánto sabían sobre el tema los médicos postulantes a la residencia. Así, a continuación se presenta la frecuencia de las respuestas por cada pregunta.

En la pregunta 1 (tabla 1) "La obtención del consentimiento informado en el campo de la salud intenta...", si bien más de la mitad (57\%) respondió b) "mejorar la calidad ética de las relaciones clínicas", uno de cada tres (37\%) eligió a) "proteger legalmente a los profesionales de la salud" (37\%). Este resultado indicaría que los participantes reconocen aspectos éticos y legales en el consentimiento. Comentaron sobre el estudio 115 participantes (7,7\% de 1 486). Las características de este grupo fueron similares al total de participantes en: edad promedio 31 años (DE 5,5), procedentes de la UNMSM

Tabla 1. Objetivo del consentimiento informado y porcentajes de respuestas.

1) La obtención del consentimiento informado en el campo de la salud intenta:

a) Proteger legalmente a los profesionales de la salud

36,5

b) Mejorar la calidad ética de las relaciones clínicas

56,6

c) Promover la equidad en la atención de salud

d) Dar prioridad de la atención de salud a los más necesitados

6,3

0,5

2) Respetar las preferencias (autonomía) del paciente significa:

a) Considerar que el paciente es incapaz de tomar decisiones de salud $\quad 2,5$

b) Considerar que el paciente no tiene información técnica 3,8

c) Mejorar la comunicación entre el médico y el paciente $\quad 84,2$

\begin{tabular}{lr} 
d) Evitar causarle angustia al paciente $\quad 9,5$ \\
\hline
\end{tabular} 
Tabla 2. Información en el consentimiento informado y porcentajes de respuestas.

3) La información en la obtención del consentimiento debe ser aquella que:

a) Daría un médico razonable y prudente

26,7

b) Necesita cada paciente en forma particular

25,3

c) Un paciente razonable necesita para tomar decisiones racionales

d) La familia exige para su paciente

46,8

7) La obligación ética y legal de proporcionar información es mayor en:

a) Situación de emergencia

22,7

b) Tratamiento electivo

12,4

c) Tratamiento experimental

63,8

d) Tratamiento de mantenimiento

8) Se justifica éticamente ocultar el diagnóstico y el tratamiento cuando:

a) El paciente tiene riesgo suicida

b) El paciente rechaza tratamiento

c) Lo solicita la familia

d) Lo solicita el juez y difundirse a los pacientes, pues tienen todo el derecho de conocer todo el procedimiento que se les realiza”.

- "El consentimiento informado es un derecho del paciente y un deber del médico, que permite proporcionar herramientas para que el paciente pueda participar en el tratamiento. Un hecho real es que el médico puede "manejar" dicho consentimiento, más por un acto paternalista”.

En la pregunta 3 (tabla 2) "La información en la obtención del CI debe ser", si bien la respuesta correcta en la perspectiva ética era b) "necesita cada paciente en forma particular", desde el punto de vista legal pueden ser la a) "daría un médico razonable y prudente" y la c) "un paciente razonable necesita para tomar decisiones racionales". Textos clásicos, como el de Faden y Beauchamp, señalan tres estándares de información que deben proporcionar los médicos: 1) Estándar del médico razonable; 2) Estándar de la persona razonable; y, 3) Estándar subjetivo ${ }^{(4)}$. La respuesta aquella que "un paciente razonable necesita para tomar decisiones racionales" (47\%) constituye el estándar de información contemplado en la legislación de países de habla inglesa. En el Perú, la Ley general de salud solo menciona en términos genéricos el derecho del paciente a la información ${ }^{(9)}$.
Acertaron dos de cada tres en las preguntas 7: c) "tratamiento experimental" (64\%) y 8 a) "riesgo suicida" (61\%), respectivamente. La Ley general de salud menciona explícitamente "a ser informado sobre la condición experimental de la aplicación de medicamentos o tratamientos" ${ }^{(9)}$. Tabla 2.

Ocultar información cuando hay riesgo suicida alude a la práctica de "la mentira piadosa" en medicina, una excepción a la norma de obtener el consentimiento informado. Un hallazgo a destacar es que uno de cada cinco $(21 \%)$ respondió que se justifica éticamente ocultar el diagnóstico y el tratamiento cuando "lo solicita la familia". Esto indicaría que la familia en nuestro medio

participa activamente en el proceso de información al paciente.

En la pregunta 4 (tabla 3), el fraseo negativo 'dificultaría' la participación del paciente en la toma de decisión médica, explicaría la baja frecuencia de aciertos: "dar toda la información médica posible" (28\%). Esta deficiencia en el instrumento fue motivo de comentarios, por ejemplo, que la pregunta era ambigua, sesgada $y$ poco precisa.

La pregunta 9 (tabla 3) explora qué hacer cuando el paciente rechaza el consentimiento informado, pese a la situación de vida o muerte. Llama la atención la frecuencia baja de las posibles respuestas correctas, "que se acoge a la excepción" (19\%) o "busca decisión sustituta" (18\%); asimismo, que el 56\% respondiera que "pide apoyo al comité de ética asistencial", cuando este tipo de comité solo tiene función consultiva y, además, en nuestro país son pocas las instituciones de salud que cuentan con este tipo de comités.

La pregunta 5 (tabla 4), de naturaleza conceptual, sobre el fundamento del consentimiento informado, tuvo una frecuencia de aciertos alta (74\%).

La pregunta 6 (tabla 5) explora quién determina la capacidad mental (competencia) del paciente para tomar decisiones de salud. Respondió correctamente a) "el médico tratante" 47\%; sin embargo, llama la atención que respondieran c) "el psiquiatra" (38\%), porque como dice Simón: "la evaluación de la capacidad de un sujeto no se reduce a hacer un diagnóstico mental. De lo que se trata es de

Tabla 3. Decisión y consentimiento informado y porcentajes de respuestas.

4) ¿Cuál de las siguientes dificultaría la participación del paciente en la toma de decisión médica?:
a) Preparar a los profesionales de la salud para el proceso del consentimiento
7,0
b) Evaluar la comprensión de la información del paciente
56,5
c) Establecer una buena relación médico paciente
d) Dar toda la información médica posible
8,7
27,8

9) Si el consentimiento informado es rechazado, pese a la situación de vida o muerte:

$\begin{array}{ll}\text { a) Busco una decisión sustituta } & 17.7\end{array}$

b) Busca acogerse a la excepción del consentimiento informado (implícito) $\quad 18,8$

c) Pido apoyo del comité de ética asistencial

d) Evito procedimiento 
Tabla 4. Fundamento del consentimiento informado y porcentajes de respuestas.

5) El consentimiento informado promueve dos valores: el bienestar personal y la autodeterminación; por consiguiente, su realización es en esencia:

a) Un imperativo legal $\quad 15,1$

b) Un imperativo ético $\quad 74,3$

c) Una norma institucional $\quad 6,8$

d) Una virtud $\quad 3,8$

Tabla 5. Capacidad mental y porcentajes de respuestas.

6) La capacidad mental (competencia) del paciente para tomar decisiones de salud lo establece:
a) El médico tratante
47,6
b) El juez
12,9
c) El psiquiatra
38,3
d) El psicólogo
1,3

averiguar si su estado mental le permite comprender la información, valorarla, sopesarla y procesarla para finalmente tomar una decisión" (3).

Los siguientes comentarios aluden al requisito de comprensión de la información:

- "El consentimiento informado puede ser tomado cuando el paciente y su familia o representante legal entiendan la situación actual de vida o riesgo del paciente".

- "El consentimiento informado debe ser considerado necesario para la atención de todo paciente que está en uso de sus facultades".

Si bien tres de cada cuatro participantes reconocen el fundamento ético del consentimiento (pregunta 5), los siguientes comentarios señalan las dificultades en su obtención:

- "Los consentimientos no son adecuados, ya que no los hacen específicos, de acuerdo a cada intervención, siendo esto en la mayoría de hospitales, muy generales".

- "Hay que incrementar el nivel cultural en las poblaciones del país. Un mejor manejo antropológico para evitar choques culturales".

\section{DISCUSIÓN}

- "Importante lo del consentimiento informado, pero muchas veces tenemos que lidiar con consentimientos preestablecidos o idiosincrasia, religión. La función médica es hacer comprender sobre la enfermedad al paciente. Establecer el vínculo de confianza”.

- "El 75\% de peruanos son analfabetos funcionales y ¿qué sentido tiene informarles?".

Un último comentario alude a la habilidad de comunicación:

- "Creo yo que todos (como humanos que somos) estamos en el derecho de conocer qué patología nos aqueja y cómo tratarla. Tal vez lo que podríamos cambiar es el hecho de ¿cómo lo decimos?, en vez de no decirlo u ocultarlo".

La principal limitación del estudio es la confiabilidad baja del instrumento (alfa de Cronbach 0,235). Esta se debe, según Goode y Hatt, a lo siguiente: a) diferencias de conocimiento; b) heterogeneidad de los sujetos; y, c) redacción de la pregunta ${ }^{(25)}$.

Respecto a la heterogeneidad de los sujetos, efectivamente, nuestra población fue muy heterogénea en su composición. El rango de edad fluctuó entre los 23 y 58 años y fueron egresados de 26 escuelas de medicina del país y 14 del exterior. Sin embargo, consideramos que esta característica de los participantes nos proporcionó una amplia información sobre conocimiento del consentimiento en el país.

Respecto a la redacción de la pregunta como factor de baja confiabilidad, creemos que pone en evidencia la dificultad de elaborar un examen de conocimiento objetivo en el campo de la ética, a diferencia de otras materias en la educación médica.

Los participantes en el estudio reconocen aspectos éticos y legales en el consentimiento. Berg y col. señalan que el consentimiento informado comprende: a) normas legales que prescriben la conducta de médicos en su relación con pacientes; b) una doctrina ética apoyada en el valor social de la autonomía, que promueve el derecho del paciente a la autodeterminación respecto a su tratamiento; c) un proceso interpersonal en el que interactúan las partes para elegir un adecuado curso de tratamiento médico. El consentimiento es todo esto y ninguno de ellos por sí solos ${ }^{(26)}$.

El consentimiento informado, institucionalizado a través del sistema judicial y sus mecanismos externos de sanción, no garantizan, necesariamente, que los médicos lo incorporarán en su práctica clínica. Aceptar el consentimiento por sus implicancias legales no beneficia a la relación médico paciente. Sin embargo, el peso de lo legal sobre lo ético se debería, según Etxeberría, a que las normas jurídicas: a) tienen mecanismos externos de sanción; b) son constitutivas de una praxis social institucionalizada; y, c) en Derecho lo que cuenta es el cumplimiento material de la norma jurídica ${ }^{(27)}$.

En nuestro estudio, solo uno de cada cuatro participantes respondió que la información debe ser aquella que necesita cada paciente en forma particular. La bioética norteamericana dice Simón que "ha entendido prácticamente desde sus inicios, que desde el punto de vista ético el estándar de información solo podría ser un estándar personalizado, esto 
es, un estándar ajustado a las peculiaridades y necesidades de cada paciente y no un estándar de información prefijada previamente, sea desde la perspectiva profesional sea desde la perspectiva del paciente razonable" (3).

En nuestro estudio casi la mitad de los participantes (47\%) respondió que el estándar de información es el de 'la persona razonable'. Tal como se mencionó en los resultados del estudio, en nuestro país, la Ley general de salud solo menciona en términos generales el derecho del paciente a la información ${ }^{(9)}$.

Respecto a una 'persona razonable', Doyal dice: "debe interpretarse como aquello que los mismos clínicos desearían saber en circunstancias similares o querrían que se informe a sus familiares o amigos cercanos... La investigación empírica sugiere que el nivel de comprensión de los pacientes mejora con la información, elaborada de acuerdo a sus necesidades y con el uso de una variedad de estrategias de buena comunicación. Y, no es sorprendente que la pobre comunicación pueda llevar hacia una pobre comprensión" (28).

En Australia e Inglaterra, las cortes han empezado a aplicar un estándar sólido a la información que los médicos están obligados a dar a sus pacientes, dicen Skene y Smallwood, aquella que un paciente razonable esperaría, más que la que un grupo de médicos razonables podrían considerar adecuado. La corte superior de Australia considera importante los siguientes factores para decidir si un riesgo es material y debe ser mencionado al paciente:

- Naturaleza del material a revelar. Se debe revelar los daños más severos y probables que ocurran.

- Naturaleza del procedimiento propuesto. Las intervenciones complejas así como los procedimientos en sujetos sanos requieren de mayor información.

- Deseo del paciente de información. Pacientes que hacen preguntas dan a conocer su deseo de información y esta debe darse.

- Temperamento y salud del paciente. Pueden necesitar mayor información pacientes ansiosos y aquellos con problemas de salud y otras circunstancias relevantes que hacen que el riesgo sea más importante en ellos (tales como su condición médica u ocupación).

- Circunstancias generales. La información necesaria para procedimientos electivos, donde es posible varias consultas, puede ser diferente de aquella requerida en un servicio de emergencia ${ }^{(21)}$.

En India, dice Arun Bal que el consentimiento fue prácticamente inexistente hasta que el Acta de protección al consumidor se hizo aplicable a la profesión médica. Ahora, tanto médicos como pacientes están más atentos a este concepto y los pacientes están mejor informados sobre sus derechos. La comunicación es el alma del CI; sin embargo, ella no se enseña a estudiantes de medicina. En India, la gran mayoría de quejas recibidas por la asociación de consumidores se presenta luego de ruptura de la comunicación médico paciente ${ }^{(29)}$.

En Canadá, Etchells y col. señalan que los estudios empíricos sugieren que el deseo del paciente de información coincide estrechamente con el estándar legal de 'persona razonable', en especial los casos quirúrgicos... Asimismo hallaron que la información verbal rutinaria no es del todo efectiva, mientras que la escrita o combinada escrita y verbal mejoran la comprensión del paciente ${ }^{(30)}$.

Simón dice que el verdadero contenido sustantivo del consentimiento informado es un proceso deliberativo cuyo resultado es una toma de decisión conjunta entre paciente y médico. El médico no decide 'por' el paciente, como hacía el paternalismo, pero el paciente tampoco debe decidir 'sin' el médico sino 'junto' al médico y 'con' el médico. Una de las mayores tragedias de la teoría del consentimiento es haber transmitido la idea de que lo fundamental era satisfacer el "derecho del paciente a ser dejado solo" frente a las decisiones sanitarias... (3).

Tanto el Derecho como la bioética norteamericana se han planteado clásicamente cinco situaciones excepcionales en las que se considera legítimo actuar sin obtener el CI de los pacientes e, incluso, a pesar de la oposición expresa de estos. Estas excepciones son: 1) Grave peligro para la salud pública; 2) Urgencia vital; 3) Incapacidad del paciente para consentir; 4) Rechazo de la información por el paciente; 5) Privilegio terapéutico ${ }^{(3)}$.

Con relación al consentimiento informado y capacidad mental, en nuestro estudio, casi la mitad de los participantes respondió que la capacidad mental lo determina el médico tratante. En el proceso de obtención del consentimiento es importante evaluar la capacidad mental del paciente. Como dice Simón: "todo médico debe asegurarse que su paciente es capaz de participar adecuadamente en el proceso de toma de decisiones. Es una responsabilidad suya que, en principio, no puede ser delegada -aunque sí compartida. Esto exige, obviamente, que todos los profesionales sanitarios reciban un mínimo de entrenamiento en este campo" (3). Sin embargo, determinar la capacidad mental no es una tarea fácil, como dicen Vargas-Parada y col., "El problema central de la competencia reside en cómo se determina esta de manera adecuada, pues la mayoría de las estrategias para definirla se basa en habilidades mentales relacionadas con los atributos de una persona autónoma, como son ciertas habilidades cognitivas y la independencia en la toma de decisiones" ${ }^{(5)}$.

Por su lado, Paterick y col. definen: "'Capacidad' significa habilidad para procesar la información recibida y comunicarse a través de respuestas significativas. Un elemento de la capacidad es que quien toma la decisión es un adulto y no ha sido declarado incompetente, $o$ inhabilitado legalmente para ejercer su derecho a la autodeterminación. 'Capacidad para tomar decisiones' significa habilidad para comprender los beneficios y riesgos significativos, las alternativas, para tomar y comunicar una decisión en el cuidado de su salud" (31). Etchells y col. señalan que: "La capacidad o capacidad para tomar una decisión es definida como habilidad en comprender información relevante para una decisión y apreciar las consecuencias razonablemente previsibles de esa decisión o la falta de ella. La capacidad es específica para decisiones particulares, p. ej., una persona puede ser capaz de decidir sobre su lugar de 
residencia, pero incapaz de decidir sobre su tratamiento médico. La capacidad también puede variar con el tiempo, $\mathrm{p}$. ej., una persona puede ser temporalmente incapaz por cuadro de delirio para luego recuperar la capacidad" (32).

En el reino Unido, los Estados Unidos y Canadá, cuando un paciente rechaza tratamiento médico, Buchanan señala que la ley establece que sus deseos expresos se respeten, a menos que se pueda demostrar que es legalmente incompetente ${ }^{(33)}$. En el Perú, la modificatoria a la Ley general de salud del 2009, en su artículo 15.2 Acceso a la información establece en el inciso g) "A ser informada sobre su derecho a negarse a recibir o continuar el tratamiento y a que se le explique las consecuencias de esta negativa. La negativa a recibir tratamiento puede expresarse anticipadamente, una vez conocido el plan terapéutico contra la enfermedad" (9).

Los resultados del presente estudio señalan claramente deficiencias de formación en conocimiento sobre el consentimiento informado. Ello explicaría parcialmente las dificultades para su plena incorporación en la práctica médica. La Ley General de Salud, vigente desde 1997, establece su obligatoriedad; sin embargo, más que por razones legales, debemos buscar que sea el trasfondo ético de esta nueva forma de relación médico paciente lo que fortalezca su uso desde las etapas formativas del profesional médico.

\section{AGRADECIMIENTO}

Al Dr. Ernesto Raez Gonzáles, Director de la Unidad de Postgrado de la Facultad de Medicina, UNMSM, y a su equipo de colaboradores. Asimismo, a todos los médicos que colaboraron y permitieron la realización del presente estudio.

\section{REFERENCIAS BIBLIOGRÁFICAS}

1. Sánchez García J. La medicina griega en su época de oro. Lima: Editorial Línea y punto SAC; 2003.

2. Gracia D. La tradición médica y el criterio del bien del enfermo. El paternalismo médico. En: Gracia D (editor). Fundamentos de bioética. Madrid: Editorial EUDEMA; 1989. p. 23-107.
3. Simón Lorda P. El consentimiento informado. Historia, teoría y práctica. 1ra edición. Madrid: Tricastela; 2000.

4. Faden RR, Beauchamp TL. A History and Theory of Informed Consent. New York: Oxford University Press; 1986.

5. Vargas-Parada L, Flisser A, Kawa S. Consentimiento informado. En: Pérez-Tamayo R, Lisker R, Tapia R [coordinadores]. La construcción de la bioética. México: Fondo de Cultura Económica; 2007.

6. Gracia D. El método de la bioética. En: Gracia D. Fundamentos de bioética. Madrid: Editorial EUDEMA; 1989. p. 395-514.

7. President's Commission for the Study of Ethical Problems in Medicine and Biomedical and Behavioral Research. Making health care decisions: the ethical and legal implications of informed consent in the patient-practitioner relationship. Washington, DC: US Government Printing Office; 1982. p. 181-3.

8. Congreso de la República. Ley General de Salud N 26842. El Peruano 20 de julio de 1997.

9. Congreso de la República. Ley que establece los derechos de las personas usuarias de los servicios de salud. Ley N 29414. El Peruano octubre del 2009.

10. Colegio Médico del Perú. Código de ética y deontología. Lima: CMP; 2007.

11. Costa MV. El manejo de la información médica: el consentimiento informado y la confidencialidad. En: Luna F, Salles ALF (eds). Bioética: nuevas reflexiones sobre debates clásicos. Buenos Aires: Fondo de Cultura Económica; 2008.

12. Braddock KCh, Edwards KA, Hasenberg NM, Laidley TL, Levinson W. Informed decision caking in outpatient practice. Time to get back to basics. JAMA. 1999;282:2313-20.

13. Pellegrino ED, Hart RJ, Henderson SR, Loeb SE, Edwards G. Relevance and utility of courses in medical ethics. JAMA. 1985;253:49-53.

14. Culver ChM, Clouser KD, Pert B, Brody H, Fletcher J, Jonson A, et al. Special report. Basic curricular goals in medical ethics. N Engl J Med. 1985;312(4):253-6.

15. Consensus statement by teachers of medical ethics and law in UK medical schools. Teaching and assessing ethics and law within medical education: a model for the UK core curriculum. J Med Ethics. 1998;24:188-92.

16. A working Group on Behalf of the Association of Teachers of Ethics and Law in Australin and New Zealand Medical School (ATEAM). An ethics core curriculum for Australian Medical School. MJA. 2001;175:205-10.

17.Zavala S, Sogi C. Evaluación de la formación ética en internos de medicina 2003.Universidad Nacional Mayor de San Marcos. An Fac med. 2006;68(1):5-18.

18. Revilla DE, Fuentes DJ. La realidad del consentimiento informado en la práctica médica peruana. Acta Med Per. 2007;24(3):223-8.
19. Sogi C, Zavala S, Ortíz P. ¿Se puede medir el aprendizaje de la ética médica? An Fac med. 2005;66(2):174-85.

20. Fisher-Jeffes L, Barton Ch, Finlay F. Clinicians knowledge of informed consent. J Med Ethics. 2007;33:181-4.

21. Skene L, Smallwood R. Informed consent: lessons from Australia. BMJ. 2002;324:39-41.

22. Roberts LW, Geppert CMA, Warner TD, Green Hammond KA, Laamberton LP. Bioethics principles, informed consent, and ethical care for special populations: curricular needs expressed by men and women physicians in training. Psychosomatics. 2005;46(5):440-50.

23. Bremberg S, Nilstun T. Patients' autonomy and medical benefit: ethical reasoning among GPs. Fam Pract. 2000;17:124-8.

24. Aiken LR. Three coefficients for analyzing the reliability and validity of ratings. Educational and psychological measurement. 1985;45:131-42.

25. Goode WJ, Hatt PK. Métodos de investigación social. México: Editorial Trillas; 1988.

26. Berg JW, Appelbaum PS, Lidz ChW, Parker LS. Informed consent. Legal theory and clinical practice. 2th edition. New York: Oxford University Press; 2001.

27. Etxeberría X. Temas básicos de ética. Ética de las profesiones. Bilbao: Editorial Desilée; 2002:8593;131-8.

28. Doyal L. Good clinical practice and informed consent are inseparable. Heart. 2002;87:103-6.

29. Bal A. "Show me the medicines". Indian J Med Ethics. 2009;6(3):170.

30. Etchells E, Sharpe G, Burgess MM, Singer PA. Bioethics for clinicians: 2. Disclosure. Can Med Assoc J. 1996;155:387-91.

31. Peterick TJ, Carson GV, Allen MC, Paterick TE. Medical informed consent: general considerations for physicians. Mayo Clinic Proc. 2008;83(3):313-9.

32. Etchells E, Sharpe G, Eliott C, Singer PA. Bioethics for clinicians: 3. Capacity. Can Med Assoc J. 1996;155:657-61.

33. Buchanan A. Mental capacities, legal competence and consent to treatment. J R Soc Med. 2004;97:415-20.

Manuscrito recibido el 16 de mayo de 2010 y aceptado para publicación el 10 de junio de 2010.

Correspondencia:

Salomón Zavala Sarrio

Instituto de Ética en Salud

Facultad de Medicina, UNMSM.

Av Grau 755. Lima 1, Perú.

Correo-e: szavalas@gmail.com 